\title{
Análisis y caracterización de señales eléctricas del cerebro utilizando interfaz cerebro computador
}

\section{Analysis and characterization of electrical signals of the brain using computer brain interface}

\author{
Emmanuel Batista ${ }^{1 *}$, Sofía Serracin ${ }^{l}$, Luis Gómez ${ }^{l}$, Rodney Moreno ${ }^{1}$, Iveth Moreno ${ }^{2}$ \\ ${ }^{1}$ Licenciatura en Ingeniería Electromecánica, Centro Regional de Chiriquí- Universidad Tecnológica de Panamá \\ ${ }^{2}$ Facultad de Ingeniería Eléctrica, Centro Regional de Chiriquí - Universidad Tecnológica de Panamá
}

\begin{abstract}
Resumen Los diferentes procesos de la actividad cerebral pueden percibirse como señales eléctricas. Algunas de ellas pueden generar potenciales en el cuero cabelludo. Estos potenciales pueden ser captados mediante un sistema de Electroencefalograma (EEG). En el caso de actividad motora (relacionada a movimientos de brazos, piernas y otros) se presenta actividad en las regiones motoras y sensoriales conocidas como ritmos sensoriomotores (SMR) en las bandas de frecuencia mu $(8 \mathrm{a} 12 \mathrm{~Hz})$ y de una parte de la banda Beta (de 18 a $30 \mathrm{~Hz}$ ), incluso, si es solo la imaginación del movimiento. Con esto en cuenta, el siguiente artículo busca la diferenciación e identificación de la intención del movimiento (imaginación motora), en base a los cambios de energía en las bandas de energía previamente mencionados y análisis topográfico, para establecer si esta actividad eléctrica es viable para generar outputs en sistemas de control a partir de ella.
\end{abstract}

Palabras clave BCI, imaginación motora, interfaz cerebro computador, ritmos sensoriomotores, SMR.

\begin{abstract}
Different processes of cerebral activity can be perceived as electrical signals. Some of them can generate potentials over the scalp. These potentials can be captured through an electroencephalogram (EEG) system. In case it's motor activity (related to arm, legs and other limb movements), regions associated with motor and sensorial tasks present a variation in activity, called sensorimotor rhythms (SMR), and is observed in the mu $(8-12 \mathrm{~Hz})$ and part of Beta $(18-30 \mathrm{~Hz})$ band frequencies, even if it's only the movement imagination. The next article looks for the differentiation and identification of the movement intention (motor imagination), in base to the changes in energy of the bands previously mentioned and topographical analysis, to establish if this electrical activity is viable to generate outputs in control systems.
\end{abstract}

Keywords BCI, motor imagination, brain computer interface, sensorimotor rhythms, SMR.

* Corresponding author: emmauel.batista@utp.ac.pa

\section{Introducción}

El cerebro en su actividad genera múltiples señales asociadas a procesos específicos (i.e. funciones diferentes) dependiendo de la región de generación [1], señales que pueden proyectarse como voltajes en el cuero cabelludo y que transmiten la intención del usuario [2]. Esta energía eléctrica puede ser registrada por un sistema de electroencefalograma (EEG). Este es el sistema de registro más utilizado en los sistemas de interfaz cerebro-computador (BCI, brain computer interface), no solo debido a que es de carácter no invasivo, sino también por ser menos costoso y más sencillo de implementar que otros [2]. Un sistema BCI, se define como un sistema en el que se crea una interfaz a partir de un elemento que adquiere señales eléctricas del cerebro (e.g. EEG, ECoG, LFP, MEG u otras [2] [3] [4]) y un computador que mediante diferentes algoritmos procesa y traduce dichas señales para ser utilizadas como comandos de control en tiempo real en distintas aplicaciones [5]. La actividad generada por las distintas tareas mentales de un individuo tiene patrones dependiendo del tipo de proceso, que pueden ser identificados (i.e. se les puede extraer características) realizando análisis en diferentes dominios como el tiempo y la frecuencia, para encontrar relaciones como tiempos de ocurrencia de eventos específicos en la señal o una secuencia de estos eventos, entre otras características. Esto implica una gran cantidad de cálculos y, por ende, mucho tiempo de proceso en las computadoras (i.e. procesador). 
Por esta razón el siguiente estudio explora la posibilidad de identificar actividad asociada a imaginación motora por medio del análisis de los ritmos sensoriomotores (SMR), los cuales están asociados a tareas motoras, buscando utilizar el promedio de la diferencia global de potenciales o energía acumulada en las bandas de frecuencia alfa y beta en $\mu \mathrm{V}^{2}$ a intervalos discretos (e.g. 1-3 segundos) como característica, en conjunto con la identificación de las regiones de generación de potenciales, mediante el uso del sistema BCI de EEG Cyton de OpenBCI de 8 canales [6].

\subsection{Los ritmos sensoriomotores (SMR)}

Los ritmos sensoriomotores son señales oscilatorias en la corteza asociadas a procesos de actividad motora y sensorial como el movimiento o la imaginación del movimiento y se observa en ritmos mu $(8-12 \mathrm{~Hz})$ y beta $(18-30 \mathrm{~Hz})$. De forma general, cuando ocurre imaginación motora de un miembro (como un brazo) se refleja la actividad sensoriomotora en el hemisferio contralateral al miembro de interés. Esta actividad se manifiesta como un ERD (Desincronización relacionada a evento) [7], que es una disminución en la energía a una banda de frecuencia determinada en la posición específica de generación. Dentro de los ritmos $m u$ los SMR tienen dos comportamientos para tener en cuenta, en donde presenta algunas variaciones en la actividad. De $8-10 \mathrm{~Hz}$ ocurre ante cualquier clase de comportamiento motor, y de $10-13 \mathrm{~Hz}$ la actividad ERD se restringe topográficamente a la zona de generación, mientras que dicha zona de generación es rodeada de ERS (Sincronización relacionada a evento), que contrario al ERD, es un aumento en el nivel de energía en la señal. En la banda Beta, se presenta igualmente ERD, pero posteriormente se observa actividad ERS [8], [9].

\subsection{El sistema Cyton OpenBCI}

El sistema Cyton de OpenBCI es una placa open-source diseñada con el fin de poder registrar actividad eléctrica corporal (e.g. actividad electroencefalográfica [EEG], eléctrica muscular o electromiográfica [EMG] y cardiaca [ECG]) para fines de demostración, desarrollo ingenieril o investigación [6] y [10]. En su aplicación para EEG el sistema registra potenciales eléctricos reflejados en el cuero cabelludo a partir de ocho electrodos sensores que se posicionan en diferentes regiones de generación de la cabeza. El sistema incluye una serie de softwares que permiten almacenar y procesar muestras, y configurar filtros y graficar las señales de cada electrodo de forma que pueda estudiarse esta actividad eléctrica [11]. Además, posee múltiples herramientas que permiten funciones como crear mapas topográficos de las señales (i.e. mapas de distribución de potenciales), determinar estados de concentración, entre otras funciones.

\section{Métodos}

\subsection{Sujetos de estudio}

Los sujetos seleccionados son tres individuos sin ninguna función motora o cerebral comprometida. Son adultos sanos pertenecientes a un proyecto de Interfaz Cerebro Computador.

\subsection{Adquisición de data y procesamiento}

La adquisición de los datos se realiza utilizando el equipo EEG de BCI de oho canales de OpenBCI. Estos canales comprenden los electrodos FP1, FP2, C3, C4, P7, P8, O1 y O2, siguiendo el sistema 10 - 20 de la convención internacional [12]. Tras la adquisición de los datos, todos los datos relativos a un proceso específico asignado, son promediados y se obtienen sus desviaciones estándar.

\subsection{Diseño del experimento y tarea}

El sujeto se sienta en una posición ergonómica en una silla (figura 1), y se calibra inicialmente el sistema, de forma que los electrodos queden en contacto con el cuero cabelludo y se le aplica un filtro rechaza banda a las señales de los sensores, mediante el software de OpenBCI para extraer la interferencia de las señales de $60 \mathrm{~Hz}$ de la línea de alimentación. El usuario se sitúa frente a una pantalla sin moverse y realiza las indicaciones que se le indican en la pantalla por medio de símbolos, palabras y sonidos. Son dos videos, uno diseñado para realizar imaginación motora del levantamiento extendido del brazo izquierdo y otro video para el brazo derecho. La secuencia de instrucciones es la siguiente:

Inicialmente hay tres segundos de espera en que no se hace nada, donde se inicializa el experimento con imagen en la pantalla "Inicio del experimento", proceden tres segundos de descanso en los cuales el sujeto cesa cualquier actividad consciente mental, con la palabra en pantalla "Descanso". Le siguen tres segundos de imaginación del movimiento (brazo izquierdo o derecho dependiendo del video), el cual se muestra en la pantalla como una flecha en la dirección del brazo que debe moverse. Posteriormente tres segundos de parpadeo, indicados con la palabra "Parpadeo". La secuencia activa (trial) de tres segundos para descanso, tres segundos para imaginación de movimiento y tres segundos de parpadeo anteriormente mencionada, se repite tres veces. Luego de tres repeticiones de dicha secuencia se cierran los ojos diez segundos para descansar la vista (en caso de personas que se le resequen rápido los ojos), mediante la orden en pantalla "Cierre los ojos", al término de los diez segundos suena un pitido grave y de bajo volumen, que indica al usuario que abra los ojos. Se repite nuevamente la secuencia activa tres veces y ocurre otro período de diez segundos de descanso de la vista. Finalmente, la secuencia activa vuelve a ocurrir, pero solo una vez, sumando un total de diez trials y tras ello se indica en la pantalla "Fin del experimento". 


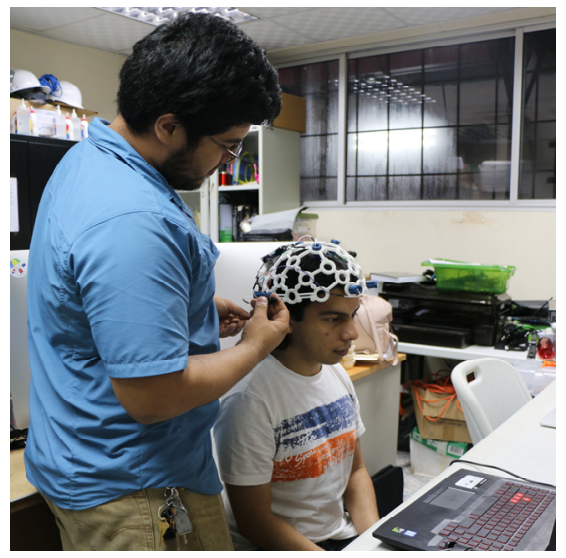

Figura 1. Calibración de los sensores EEG.

\subsection{Análisis de energía en banda de frecuencia}

Los ritmos en los cuales se refleja la actividad sensoriomotora útil para EEG son los mu, de $8-12 \mathrm{~Hz}$ y parte de los ritmos Beta de $18-30 \mathrm{~Hz}$. Teniendo esto en cuenta, se puede enfatizar en disminuciones de energía como los ERD y de esta forma identificar la imaginación de movimiento del sujeto observando los niveles de energía a tales frecuencias y su distribución topográfica de actividad entre $10-13 \mathrm{~Hz}$.

\section{Resultados}

Utilizando el sistema de OpenBCI, se obtuvieron mapas topográficos de la actividad EEG relacionada a la imaginación motora. En ellos, se observó de forma marcada el comportamiento de ERD en forma contralateral a la imaginación motora del miembro. Esto es, que en la región motora del hemisferio izquierdo cerebral se percibió un menor nivel de energía cuando se realizó imaginación del movimiento del brazo derecho (figura 2); y que en la región motora del hemisferio derecho cerebral se percibió un menor nivel de energía cuando se realizó imaginación del movimiento del brazo izquierdo (figura 3). Por otro lado, durante el intervalo de descanso se presentó simetría de potenciales entre hemisferios (figura 4).

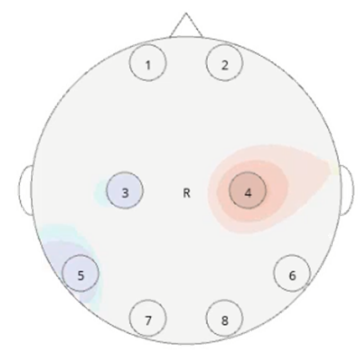

Figura 2. Mapa topográfico de la actividad: Imaginación motora, brazo derecho. Desincronización izquierda.

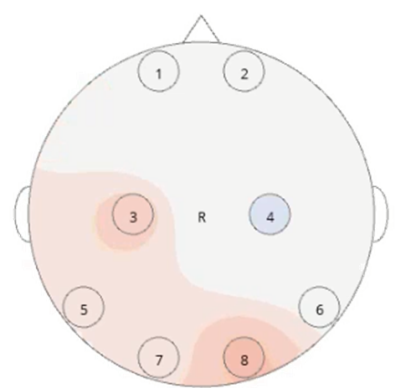

Figura 3. Mapa topográfico de la actividad: Imaginación motora brazo izquierdo. Desincronización derecha.

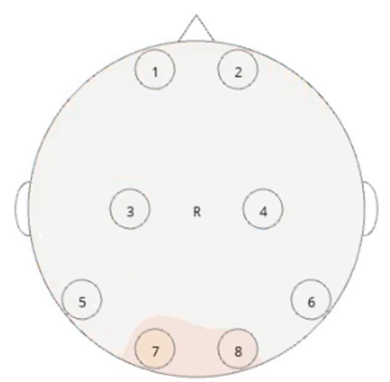

Figura 4. Mapa topográfico de la actividad: Descanso.

En cuanto al análisis en frecuencia, la energía de bandas demostró algunas diferencias esenciales entre las diferentes tareas cerebrales (descanso e imaginación motora).

En el proceso de imaginación motora, tanto el brazo izquierdo como el derecho presentaron una tendencia en la que Alfa tiene mayor nivel de Energía que Beta, como se observa en la figura $5 a$ y figura $5 b$.

Por otro lado, en la tarea de descanso se observó que la banda Beta presentaba mayor nivel de energía que la banda Alfa (figura 5c).

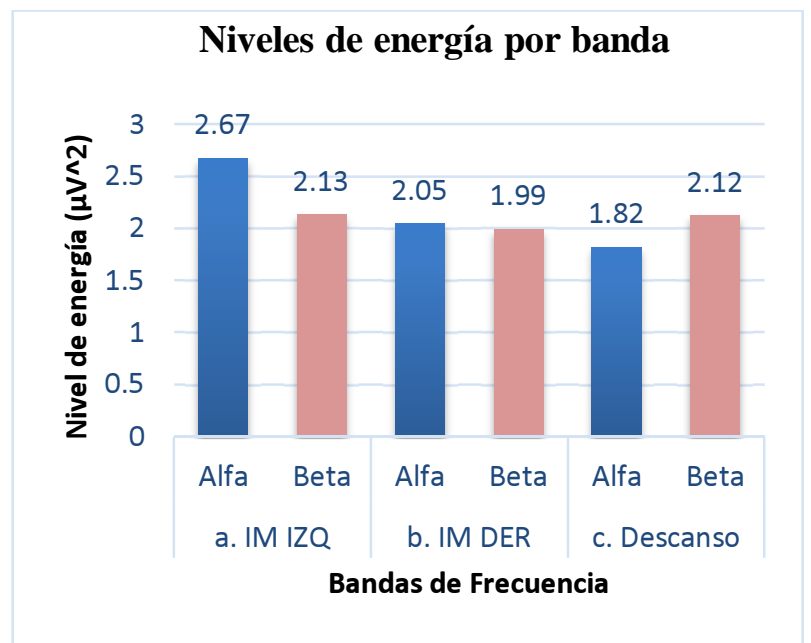

Figura 5. Niveles de energía por banda de frecuencia de: a. Brazo izquierdo. Desviación estándar en Alfa: 0.2376; Beta: 0.1068. b. Brazo derecho. Desviación estándar en Alfa: 0.2577; Beta 0.1894. c. Descanso. Desviación estándar en Alfa: 0.1206; Beta 0.1739. 


\section{Discusión}

Muchos estudios realizados en el campo BCI buscan analizar la actividad SMR a partir del uso de porcentajes de sincronización y desincronización en las energías de banda [13]. Por otro lado, para este estudio se ha buscado una alternativa que utilice los potenciales sin una normalización previa y sin el uso de porcentajes, utilizando la interfaz gráfica de OpenBCI. De esta forma se pueden ahorrar recursos del procesador.

Si bien el estudio demostró ser viable, aún se encuentra en etapa de desarrollo, por lo que se espera realizar pruebas con una población mucho mayor para desarrollar un posterior estudio para aplicaciones prácticas con imaginación motora.

\section{Conclusiones}

- Se puede diferenciar en base a los análisis topográficos en que zona del cerebro se ve reflejada mayor y menor actividad, según el proceso de imaginación motora de cada brazo, mediante comportamiento de la desincronización contralateral.

- Los niveles de energía por bandas de cada una de las tareas muestran una tendencia a mayor magnitud en la banda Alfa (respecto a la Beta) en la imaginación motora de ambos brazos; por otro lado, en el descanso se observa un mayor nivel de energía en la banda Beta (respecto a Alfa).

- El uso de los niveles de energía de bandas para asociar a outputs (comandos) de sistemas de control, solo es viable realizando de forma conjunta, un análisis topográfico del comportamiento de los ERDs.

\section{AGRADECIMIENTO}

Estos estudios se realizan gracias a financiamiento de la SENACYT, dentro de la convocatoria I+D 2015 del proyecto BCI N ${ }^{\circ}$ 86-2016-4IDDS15-0994, "Desarrollo de un sistema domótico basado en una interfaz cerebro-maquina para dar soporte a personas con movilidad reducida".

\section{Referencias}

[1] L. E. Miller y N. Hatsopoulos, «Neuronal Activity in Motor Cortex and Related Areas,» de BCI Principles and Practices, New York, Oxford University Press, 2012, p. 15.

P. L. Nunez, «Electric and Magnetic Fields Produced by the Brain,» de BCI Principles and Practices, New York, Oxford University Press, 2012, pp. 45-46.

[3] R. Kristeva-Feige, C. Fritsch y J. T. Carl-HermanLücking, «Effects of attention and precition of exerted force on beta range EEG-EMG synchronization during a manteined motor contraction task,» J. Clinical Neurophysiology, vol. 113, $\mathrm{n}^{\circ}$ 1, pp. 124-131, 2002.
[4] T. Camilo, D. Günter, T. Robert, S. Susumu, K. Conrad y H. Mark, «Event-related desynchronization and movement related cortical potencials ECoG and EEG,»J. Clinical Neurophysiology, vol. 93, nº 5, pp. 380-389, 1994.

[5] D. J. McFarland y J. R. Wolpaw, «EEG-based braincomputer interfaces,» Curr. Opin Biomed, vol. 4, pp. 194200, 2017.

[6] OpenBCI, «OpenBCI Shop,» [En línea]. Available: https://shop.openbci.com/collections/frontpage/products/cy ton-biosensing-board-8-channel?variant=38958638542.

[7] G. Pfurtscheller y A. Aranibar, «Evaluation of event-related desynchronization (ERD) preceding and following voluntary self-paced movement,» Electroencephalogr. Clin. Neurophysiol., vol. 46, n 2, pp. 138-146, 1979.

[8] G. Pfurtscheller, «Central beta rhythm during sensorimotor activities in man,» Clin. Neurophysiol., vol. 51, $\mathrm{n}^{\circ} 3$, pp. 253-264, 1981

[9] C. Keinrath, S. Wriessnegger, G. R. Müller-Putz y G. Pfurtscheller, «Post-movement beta synchronization after kinesthetic illusion, active and passive movements,» Int. J. Physhophysiol., vol. 62, no 2, pp. 321-327, 2006.

[10] OpenBCI, «Open Brain Computer Interface,» [En línea]. Available: https://openbci.com/.

[11] OpenBCI, «OpenBCI Software,» [En línea]. Available: http://docs.openbci.com/OpenBCI\%20Software/00OpenBCISoftware.

[12] American Electroencephalographic Society, «American Electroencephalographic Society Guidelines for Standard Electrode Position Nomenclature,» Journal of Clinical Neurophysiology, vol. 8, n 2, pp. 200-202, 1991.

[13] M. Tariq, L. Uhlenberg, P. Trivailo, K. S. Munir, and M. Simic, "Mu-beta rhythm ERD/ERS quantification for foot motor execution and imagery tasks in BCI applications," 8th IEEE Int. Conf. Cogn. Infocommunications, CogInfoCom 2017 - Proc., vol. 2018-January, no. CogInfoCom, pp. 9196, 2018. 